\title{
Efficacy of Samsharkara Churna in Kaphaj Kasa
}

\author{
Research Article
}

\section{Prerana Madhav Dighe ${ }^{1^{*}}$}

1. Associate Professor, H.O.D, Department of Kayachikitsa, Ayurved College, Rahuri.413706. Maharashtra, India.

\begin{abstract}
According to Ayurveda kasa is an independent disease, the Excellency of ayurveda over other medical sciences is that it had not only metioned ' $\mathrm{Kasa}$ ' as a symptom in various disease but also described it as an independent vyadhi with its separate Pathogenesis, symptoms, signs, types and treatment. If control over kasa vyadhi is not achieved in time it can give rise to life threatening diseases like Swasa, Shosa, Rajyakshama, UraKshata, Rakttapitta, granthi, Arbuda of respiratory tract etc. so to avoid above complications it is must to avoid on kasa vyadhi, hence a clinical study was carried out to study efficacy of Samsharkara churna. In the present study 60 patients are studied. The result were statistically analyzed with the help of chi-square test and the result have shown that the samsharkara churna have good results in the kaphaj kasa.
\end{abstract}

Keywords: Kaphaj Kasa, samsharkara churna, Cough.

\section{Introduction:}

Prana the most important among the five types of vayu and related with life, therefore any abnormality in its function leads to disturbance of all the functions as prana is sarvagata as "Kasa is one of the primary disease of pranavaha strotasa, if neglected can affect 'Sarvagat prana' \& can hence cause disturbance in other body functions. (1)(2)(3)

Correlating modern science with ayurveda, oxygen present in air that is

The central cough suppressants e.g. opioids like codein, noscapine, dextro methorphan, and antihistaminic antitussives like diphenhydramine, steroids e.g. prednisolone are the commonest drugs and used for symptomatic relief from

*Corresponding Author:

\section{Prerana Madhav Dighe}

Associate Professor, H.O.D., PG Guide

Department of Kayachikitsa,

Ayurved College, Rahuri.413706.

Maharashtra, India

Email: dr.astro.preranadighe@gmail.com taken inside lungs during inspiration can be considered as Bahya prana vayu i.e. ambarpiyusha (4). Oxyhaemoglobin from alveo-capillary exchange and nutritional substances produced after metabolism enter the blood. Both of them constitute the internal (Aabhayantar) prana vayu as both of them nourish every tissue of body.

In modern medicine the drug, which are used in symptomatic treatment of cough, are called antitussive.

cough.

Ayurveda had not only provided a wide range of drugs but also provided the various preventive measures to be followed while cured the disease. The respiratory system may be affected by various inhaled toxic agents. These inhaled toxic agents acts by direct toxicity on respiratory system or through immune mechanism. Such exposure can be either occupational or environmental.

Ayurveda has not only described inhaled toxins but also described impairment of digestive process as one of 
the constituent of pathogenesis of kasa vyadhi.

Charak, the father of Indian medicine has mentioned basic principles of treatments for the management of kasa.

1) Shodhana Chikitsa

2) Shamana Chikitsa

Shamana is one of the easiest and economical therapy

for kasa. Churna is one of the kalpana mentioned by Sharangdhar in Sharangdhar samhita (5).

\section{Materials \& Methods:-}

In the present study Samsharkara Churna(6) will be prepared using plants given below with Bhavaprakash Samhita.

Table 1: Showing the plants used for the preparation of the Samsharkara Churna.

\begin{tabular}{|c|c|c|c|c|}
\hline $\begin{array}{l}\text { Sr. } \\
\text { no }\end{array}$ & $\begin{array}{l}\text { Drug } \\
\text { Nam } \\
\text { e }\end{array}$ & $\begin{array}{l}\text { Botanical } \\
\text { Name }\end{array}$ & Family & $\begin{array}{l}\text { Qt } \\
\text { y. }\end{array}$ \\
\hline 1 & $\begin{array}{l}\text { Lava } \\
n g\end{array}$ & $\begin{array}{l}\text { Syzygium } \\
\text { aromaticum } \\
\text { (Linn.) }\end{array}$ & $\begin{array}{l}\text { Myrtace } \\
\text { ae }\end{array}$ & $\begin{array}{l}10 \\
\mathrm{gm}\end{array}$ \\
\hline 2 & $\begin{array}{l}\text { Jatip } \\
\text { hala }\end{array}$ & $\begin{array}{l}\text { Mystrica } \\
\text { fragrance } \\
\text { (Houtt.) }\end{array}$ & $\begin{array}{l}\text { Myristi } \\
\text { ceae }\end{array}$ & $\begin{array}{l}10 \\
\mathrm{gm}\end{array}$ \\
\hline 3 & $\begin{array}{l}\text { Pippa } \\
l i\end{array}$ & $\begin{array}{l}\text { Piper } \\
\text { longum(Lin } \\
\text { n.) }\end{array}$ & $\begin{array}{l}\text { Piperac } \\
\text { eae }\end{array}$ & $\begin{array}{l}10 \\
\text { gm }\end{array}$ \\
\hline 4 & $\begin{array}{l}\text { Maric } \\
h\end{array}$ & $\begin{array}{l}\text { Piper } \\
\text { nigrum(Lin } \\
\text { n.) }\end{array}$ & $\begin{array}{l}\text { Piperac } \\
\text { eae }\end{array}$ & $\begin{array}{l}20 \\
\text { gm }\end{array}$ \\
\hline 5 & $\begin{array}{l}\text { Shunt } \\
\text { hi }\end{array}$ & $\begin{array}{l}\text { Zinziber } \\
\text { officinale( } \mathrm{R} \\
\text { oxb.) }\end{array}$ & $\begin{array}{l}\text { Zinzibe } \\
\text { raece }\end{array}$ & $\begin{array}{l}16 \\
\text { gm }\end{array}$ \\
\hline
\end{tabular}

Other constituents:-

1) Sharkara $210 \mathrm{gm}$.

Table 2:- Properties of Drug

\begin{tabular}{|l|l|l|l|}
\hline $\begin{array}{l}\text { Drug / } \\
\text { Chara } \\
\text { cter }\end{array}$ & $\begin{array}{l}\text { Marich } \\
\text { (7) }\end{array}$ & $\begin{array}{l}\text { Pippali } \\
\text { (8) }\end{array}$ & $\begin{array}{l}\text { Jaypha } \\
\text { l (9) }\end{array}$ \\
\hline Gana & $\begin{array}{l}\text { Dipaniya } \\
\text { Shirovirec } \\
\text { hana }\end{array}$ & $\begin{array}{l}\text { Kasahar } \\
\text { Hikkanig } \\
\text { raha }\end{array}$ & \\
\hline
\end{tabular}

\begin{tabular}{|c|c|c|c|}
\hline & (Cha) & (Cha) & \\
\hline Family & Piperaceae & $\begin{array}{l}\text { Piperacea } \\
\text { e }\end{array}$ & $\begin{array}{l}\text { Mytrac } \\
\text { eae }\end{array}$ \\
\hline $\begin{array}{l}\text { Latin } \\
\text { Name }\end{array}$ & $\begin{array}{l}\text { Piper } \\
\text { nigrum }\end{array}$ & $\begin{array}{l}\text { Piper } \\
\text { longum }\end{array}$ & $\begin{array}{l}\text { Myristi } \\
\text { ca } \\
\text { fragran } \\
\mathrm{S}\end{array}$ \\
\hline Guna & $\begin{array}{l}\text { Rasa- katu } \\
\text { Vipak- } \\
\text { Katu } \\
\text { Virya - } \\
\text { Ushna } \\
\text { Guna- } \\
\text { Laghu, } \\
\text { tikshna }\end{array}$ & $\begin{array}{l}\text { Rasa- } \\
\text { tikta,katu } \\
\text { Vipak- } \\
\text { Katu, } \\
\text { shushka } \\
\text { Virya - } \\
\text { Ushna } \\
\text { Guna- } \\
\text { Laghu, } \\
\text { tikshna }\end{array}$ & $\begin{array}{l}\text { Rasa- } \\
\text { tikta, } \\
\text { katu } \\
\text { Vipak- } \\
\text { Katu } \\
\text { Virya- } \\
\text { Ushna } \\
\text { Guna- } \\
\text { Laghu, } \\
\text { tikshna } \\
\text { Snigdh } \\
\text { a }\end{array}$ \\
\hline $\begin{array}{l}\text { Useful } \\
\text { part }\end{array}$ & $\begin{array}{l}\text { (Fruit) } \\
\text { Phala }\end{array}$ & Phala & $\begin{array}{l}\text { Beeja } \\
\text { (Seeds) } \\
\text { Kosha }\end{array}$ \\
\hline Dose & $\begin{array}{l}\text { Churna 1- } \\
5 \text { gms }\end{array}$ & $0.5-1 \mathrm{gms}$ & $\begin{array}{l}0.5- \\
1 \mathrm{gms}\end{array}$ \\
\hline
\end{tabular}

Table 3:-

\begin{tabular}{|c|c|c|}
\hline $\begin{array}{l}\text { Drug I } \\
\text { Characte } \\
\text { r }\end{array}$ & Lavang (10) & Suntha (11) \\
\hline Gana & Mytraceae & $\begin{array}{l}\text { Zinziberacea } \\
\mathrm{e}\end{array}$ \\
\hline Family & Piperaceae & Piperaceae \\
\hline $\begin{array}{l}\text { Latin } \\
\text { Name }\end{array}$ & $\begin{array}{l}\text { Syzygium } \\
\text { aromaticum }\end{array}$ & $\begin{array}{l}\text { Zinziber } \\
\text { officinale }\end{array}$ \\
\hline Guna & $\begin{array}{l}\text { Rasa- } \\
\text { tikta,katu } \\
\text { Vipak- Katu } \\
\text { Virya-shita } \\
\text { Guna- Laghu, } \\
\text { tikshna,snigdh } \\
\text { a }\end{array}$ & $\begin{array}{l}\text { Rasa-katu } \\
\text { Vipak- } \\
\text { madhur } \\
\text { Virya } \\
\text { Ushna } \\
\text { Guna- } \\
\text { Laghu, } \\
\text { snigdha }\end{array}$ \\
\hline $\begin{array}{l}\text { Useful } \\
\text { part }\end{array}$ & $\begin{array}{l}\text { (Flower) } \\
\text { Pushpa }\end{array}$ & $\begin{array}{l}\text { Kanda } \\
\text { (Rhizome) }\end{array}$ \\
\hline Dose & $\begin{array}{l}\text { Churna } \\
2 \text { gms }\end{array}$ & $\begin{array}{l}\text { Churna 1- } \\
2 \text { gms }\end{array}$ \\
\hline
\end{tabular}


Method:-

A) Sixty patient of kaphaj kasa vyadhi willing for treatment.

B) Samsharkara Churna was prepared in the department of kayachikitsa of S.V.N.H.T's, Ayurved college, rahuri.

60 patients fulfilling the criteria for the diagnosis of disease were registered from the OPD of Kaychikitsa Dept. of Ayurved College Hospital, Rahuri, and Samsharkara Churna was given to all of them.

Grouping

1. Trial Group - 30 patients - Trial Drug Dose - 10 gm

2. Control Group - 30 Patients Starch Capsul dose - 10 gm.

Written consent was taken from patient before starting therapy, various time to time investigation and examining procedures are done.

\section{Investigations:-}

1) Chest X-Ray

2) Erythrocyte Sedimentation Rate

3) Complete Blood Count (WBC count)

\section{Selection Criteria:-}

1) Inclusive criteria-

1) Patient ready for this study willing for treatment.

2) Patient of age group 18 to 60 years of age.

3) Patient with signs \& symptoms of Kaphaj kasa as described in grantha.(12)

4) Patient selection will be irrespective of season and prakruti also.

\section{2) Exclusive Critetria:-}

1) Patients with vataj, pittaj, kshayaj, kshataja kasa.

2) Patient with severe systematic disease like cardiac disease, renal failure, CVA, HIV etc., will be excluded.

3) Patients with pneumonia, Asthma, T.B., Bronchiectasis, CA Lungs etc will be excluded.
4) Pregnant women and lactating mothers will be excluded.

5) Patients below age of 18 Years and above age $60 \mathrm{yrs}$.

Table 4: Patient's frequency acc. to age group

\begin{tabular}{|l|l|l|}
\hline $\begin{array}{l}\text { Age } \\
\text { group in } \\
\text { years }\end{array}$ & $\begin{array}{l}\text { No. of } \\
\text { Patients(Out } \\
\text { of 60) }\end{array}$ & Percentage \\
\hline $18-25$ & 11 & $18.33 \%$ \\
\hline $26-35$ & 19 & $31.66 \%$ \\
\hline $36-45$ & 19 & $31.66 \%$ \\
\hline $46-60$ & 11 & $18.33 \%$ \\
\hline
\end{tabular}

Table 5:- Patient's Frequency acc. to sex

\begin{tabular}{|l|l|l|}
\hline $\begin{array}{l}\text { Sex of } \\
\text { patient }\end{array}$ & $\begin{array}{l}\text { No. of } \\
\text { Patient(out } \\
\text { of 60) }\end{array}$ & Percentage \\
\hline Male & 48 & $80.00 \%$ \\
\hline Female & 12 & $20.00 \%$ \\
\hline
\end{tabular}

Table 6:- Patients Frequency acc. to Prakruti

\begin{tabular}{|l|l|l|}
\hline Prakruti & $\begin{array}{l}\text { No.of } \\
\text { patient } \\
\text { (out of } \\
\text { 60) }\end{array}$ & Percentage \\
\hline $\begin{array}{l}\text { Vata Pradhan } \\
\text { Kapha }\end{array}$ & 12 & $20.00 \%$ \\
\hline $\begin{array}{l}\text { Vata Pradhan } \\
\text { Pitta }\end{array}$ & 14 & $23.33 \%$ \\
\hline $\begin{array}{l}\text { Pitta Pradhan } \\
\text { Vata }\end{array}$ & 10 & $16.67 \%$ \\
\hline $\begin{array}{l}\text { Pitta Pradhan } \\
\text { Kapha }\end{array}$ & 06 & $10.00 \%$ \\
\hline $\begin{array}{l}\text { Kapha Pradhan } \\
\text { Vata }\end{array}$ & 07 & $11.67 \%$ \\
\hline $\begin{array}{l}\text { Kapha Pradhan } \\
\text { Pitta }\end{array}$ & 11 & $18.33 \%$ \\
\hline
\end{tabular}

Table 7:-

Schedule

of

Treatment

\begin{tabular}{|l|l|}
\hline Medicine & $\begin{array}{l}\text { Samsharkara } \\
\text { Churna }\end{array}$ \\
\hline Dose & 10gms / Day, \\
\hline
\end{tabular}




\begin{tabular}{|l|l|}
\hline & divided into 3 doses. \\
\hline Anupana Sevan & $\begin{array}{l}\text { 1) Apaan kali } \\
\text { Prabhat kala } \\
\text { 2) Pratah Paschat } \\
\text { Bhakta } \\
\text { 3) Sayam paschat } \\
\text { Bhakta } \\
\text { kala. }\end{array}$ \\
\hline $\begin{array}{l}\text { Duration } \\
\text { medicine }\end{array}$ & 15 day \\
\hline Follow up & 5/10/15 Day's \\
\hline
\end{tabular}

Table 8: Observation Table

\section{A) Objective Parameters:-}

1) Frequency of cough attack in $24 \mathrm{hrs}$.

- 10 cough attack in $24 \mathrm{hrs}$ : Grade I

- 20 cough attack in $24 \mathrm{hrs}$ : Grade II

- 30 cough attack in 24hrs : Grade III

2) Duration of Each cough attack.

- 5-10 sec- Grade-I

- 15-60 sec- Grade- II

- more than 90 sec- Grade-III

3) Kshtivan

- Alpa Kshtivan- Grade I

- Madhyam Kshtivan - Grade II

- Prabhut Kshtivan - Grade III

4) Chardi Vega / Day

- Absent

- Present

- Present - number of Chardivega

5) Jwara- present, absent

6) Auscultation
A] Rhonchi-

Alpa-Low Intensity Sound- Grade I

Madhyam- High Intensity sound- Grade II

Prabhut - Very High intensity sound-

Grade III

B] Crepts-

Alpa- Low Intensity Sound-Grade I

Madhyam- High Intensity sound- Grade II

Prabhut - Very High intensity sound-

Grade III

B) subjective Parameters:-

1) Shwas Kashtata:

Absent - Grade I

Mild - Grade II

Moderate - Grade III
2) Mandgani-

-Hunger after $6 \mathrm{hrs}$ of $1^{\text {st }}$ meal in a day-

Grade I

-Hunger after 8 hrs of $1^{\text {st }}$ meal in a dayGrade II day-

-Hunger after $12 \mathrm{hrs}$ of $1^{\text {st }}$ meal in a

Grade III

3) Aruchi- Present, Absent

4) Aanggaurava-Present, Absent

5) Lomaharsha - Present, Absent

6) Aasymadhurya-Present, Absent

Table No.-9

Frequency of

Clinical features.

\begin{tabular}{|l|l|l|}
\hline $\begin{array}{l}\text { Clinical } \\
\text { Features }\end{array}$ & $\begin{array}{l}\text { No of } \\
\text { Patients } \\
\text { (out of 60) }\end{array}$ & Percentage \\
\hline $\begin{array}{l}\text { Frequency of } \\
\text { cough attack } \\
\text { in } \\
\text { 24 hrs. }\end{array}$ & 60 & 100 \\
\hline Kshtivan & 28 & 46.65 \\
\hline $\begin{array}{l}\text { Chardi vega } \\
\text { day }\end{array}$ & 3 & 5 \\
\hline Jwara & 2 & 3.33 \\
\hline Ronchi & 3 & 5 \\
\hline Crepts & 1 & 1.66 \\
\hline Shwaskshtata & 10 & 16.66 \\
\hline Mandagni & 53 & 88.33 \\
\hline Aruchi & 38 & 63.33 \\
\hline Lomaharsha & 28 & 46.65 \\
\hline Aanggaurava & 23 & 38.30 \\
\hline $\begin{array}{l}\text { Aasya } \\
\text { madhurya }\end{array}$ & 15 & 25 \\
\hline
\end{tabular}

Table No. 10

Results seen in

Clinical Features

\begin{tabular}{|l|l|l|l|}
\hline $\begin{array}{l}\text { Sr } \\
\text { no }\end{array}$ & Symptoms & $\begin{array}{l}\text { Samsharka } \\
\text { ra Churna }\end{array}$ & $\begin{array}{l}\text { Starch } \\
\text { Capsul } \\
\text { e }\end{array}$ \\
\hline 1 & $\begin{array}{l}\text { Frequency of } \\
\text { cough attack } \\
\text { in 24 hrs }\end{array}$ & $100 \%$ & $38 \%$ \\
\hline 2 & Ksthivan & $46.65 \%$ & $2 \%$ \\
\hline
\end{tabular}




\begin{tabular}{|l|l|l|l|}
\hline 3 & $\begin{array}{l}\text { Chardi vega } \\
\text { / day }\end{array}$ & $5 \%$ & $1 \%$ \\
\hline 4 & Jwara & $3.33 \%$ & $0 \%$ \\
\hline 5 & Rhonchi & $5 \%$ & $0 \%$ \\
\hline 6 & Crepts & $16.66 \%$ & $1 \%$ \\
\hline 7 & $\begin{array}{l}\text { Shwaskshtat } \\
\text { a }\end{array}$ & $16.66 \%$ & $2 \%$ \\
\hline 8 & Mandagni & $88.33 \%$ & $5 \%$ \\
\hline 9 & Aruchi & $63.33 \%$ & $6 \%$ \\
\hline 10 & Lomaharsha & $46.65 \%$ & $3 \%$ \\
\hline 11 & Aanggaurva & $38.30 \%$ & $10 \%$ \\
\hline 12 & $\begin{array}{l}\text { Aasymadhar } \\
\text { ya }\end{array}$ & $25 \%$ & $7 \%$ \\
\hline
\end{tabular}

Table No. 11 Result in clinical feature Kasa Vega Frequency in 24 Hours.

\begin{tabular}{|c|c|c|c|c|c|}
\hline $\begin{array}{l}\text { r. } \\
\text { n }\end{array}$ & $\begin{array}{l}\text { Upash } \\
\text { aya } \\
\text { /Anup } \\
\text { ashay }\end{array}$ & $\begin{array}{l}\text { No. } \\
\text { of } \\
\text { Pati } \\
\text { ents } \\
\text { (Out } \\
\text { of } \\
\text { 30) }\end{array}$ & $\begin{array}{l}\text { By } \\
\text { Samsh } \\
\text { arkara } \\
\text { Churna } \\
(\%)\end{array}$ & $\begin{array}{l}\text { No } \\
\text { of } \\
\text { Pati } \\
\text { ents }\end{array}$ & $\begin{array}{l}\text { By } \\
\text { star } \\
\text { ch } \\
\text { tabl } \\
\text { et } \\
(\%)\end{array}$ \\
\hline 1 & $\begin{array}{l}\text { Uttam } \\
\text { Upash } \\
\text { aya }\end{array}$ & 22 & $73.33 \%$ & 3 & $\begin{array}{l}10.0 \\
0 \%\end{array}$ \\
\hline 2 & $\begin{array}{l}\text { Madhy } \\
\text { am } \\
\text { Upash } \\
\text { aya }\end{array}$ & 0 & $0 \%$ & 4 & $\begin{array}{l}13.3 \\
3 \%\end{array}$ \\
\hline 3 & $\begin{array}{l}\text { Anupa } \\
\text { shaya }\end{array}$ & 8 & $26.66 \%$ & 23 & $\begin{array}{l}76.6 \\
6 \% \\
\end{array}$ \\
\hline
\end{tabular}

Above sign and symptoms (both subjective and objective parameters, as shown in table no. 8) are considered as clinical picture of kaphaj kasa vyadhi for analysis of the results obtained by medicine samsharkara churna.

Criteria for assessment of overall symptoms-

1) Cured- Total Relief In Symptoms $75 \%$ To $100 \%$

2) Markedly Improved- $50 \%$ To $75 \%$ Improvement From Signs And
Symptoms Is Termed As Markedly Improved

3) Improved- Improvement Range In Between 25\% To 50\% Responded By Patient In Signs And Symptoms Is Taken For Improved.

4) Unchanged- The Patient Presenting Less Than 25\% Improvement In Their Signs And Symptoms Are Taken As Unchanged.

Table 12:- Percentage of relief of Samsharkara Churna and Starch Capsule.

\begin{tabular}{|l|l|l|l|l|}
\hline & \multicolumn{2}{|l|}{$\begin{array}{l}\text { Samsharkara } \\
\text { Churna }\end{array}$} & \multicolumn{2}{l|}{ Starch Capsule } \\
\hline Effect & $\begin{array}{l}\text { No. } \\
\text { of } \\
\text { Patie } \\
\text { nt }\end{array}$ & $\begin{array}{l}\text { Percent } \\
\text { age }\end{array}$ & $\begin{array}{l}\text { No. } \\
\text { of } \\
\text { Patie } \\
\text { nt }\end{array}$ & $\begin{array}{l}\text { Percent } \\
\text { age }\end{array}$ \\
\hline Cured & 7 & 23.33 & 4 & 13.33 \\
\hline $\begin{array}{l}\text { Marked } \\
\text { ly } \\
\text { Improv } \\
\text { ed }\end{array}$ & 21 & 70 & 15 & 50 \\
\hline $\begin{array}{l}\text { Improv } \\
\text { ed }\end{array}$ & 2 & 6.66 & 10 & 33.33 \\
\hline $\begin{array}{l}\text { Unchan } \\
\text { ged }\end{array}$ & 0 & 0 & 1 & 3.33 \\
\hline Total & 30 & 100 & 30 & 100 \\
\hline
\end{tabular}

\section{Discussion and Conclusion-}

Sixty patients with Kaphajkasa vyadhi were studied for the efficacy of samsharkara churna In samprapti of the disease following are the etiological factors.

Dosha-vata, Pitta, Kapha, mainly apana vayu prakop which causes in turn prakop of udan and pranvayu.

Dushya- Rasa raktadi seven dhatus as they related to pranvaha strotasa.

Sthana sansrya- pranvaha strotasa and its mula sthana(13)

Strotodushti- Mainly pranavha strotasa and other strotasa i.e. Rasa, Rakta udaka, 
majja, Anna, and purishvaha strotasa are related to it.

Maximum number of patients belongs to age group 26 to 45 . Out of 60 patients were $80 \%$ male and $20 \%$ were female. Regarding prakruti $20 \%$ belong to vata pradhan kapha, $23.33 \%$ belong to vata pradhan pitta. $16.67 \%$ belong to pitta pradhan vata. $10 \%$ belong to pitta pradhan kapha. $11.67 \%$ belong to kapha pradhan vata and $18.33 \%$ belong kapha pradhan pitta prakruti.

Out of 60 patients $13.33 \%$ were students. 25 to $30 \%$ of patients showed addiction to smoking, alcohol intake and tobacco chewing.

The result obtained in the present study showed that samsharkara churna is highly effective in the management of kaphaj Kasa. Starch capsules showed only significant result. In the treatment with samsharkara Churna 23.33\% got completely cured, $70 \%$ markedly improved and $6.66 \%$ got improved, where as with the starch capsule $13.33 \%$ got completely cured, $50 \%$ got markedly improved, $33.33 \%$ have got improved and $3.33 \%$ patient didn't show any change in the condition. Thus the result has shown that the samsharkara churna is very effective against Kaphaj Kasa Vyadhi.

\section{References:-}

1) Dr. Ganesh Krushna Gorde, Sartha Vagbhat of maharshi Vagbhat, Edition -2011, Varanasi. Chaukhamba surbharati (Prakashan)-2011. Page-1

2) Prof. Dr. Y.G. Joshi, Charak samhita of Maharshi Agnivesha with charakdatta commentary, $2^{\text {nd }}$ edition, Pune, Vaidya mitra Prakashan (Acadamy),-2005. Page no-415.

3) Ambikadatta, Sushruta samhita of Acharya Sushrut Dalhancharya commentary, $19^{\text {th }}$ Edition, Varanasi, Chaukhamba Sanskrit sansthan-2009. Page No-465.
4) Dr. Smt. Shailaja Shrivastav Sharangdhar Samhita of Acharya Sharngdhar. $2^{\text {nd }}$ Edition Varanasi, Chukambha orientalia, page no. 45

5) Dr. Gangadhar Vasudevshastri Sathe, Sartha Sharangdhar Samhita, $4^{\text {th }}$ edition, Pune, Sulabha mudranalaya, 1983. Page No. 127

6) Pandit Sri Brahma Sankara Misra, Bhavprakasa of Sri Bhavamisra, $5^{\text {th }}$ Edition, Varanasi, Chaukhamba Sanskrit Sansthan 1988. Page No.-154.

7) Dr. Krushachandra Chunekar \& Dr. Gangasahay Pandeya, Bhavprakashnighantu of Sri bhavamisa, $\quad 9^{\text {th }} \quad$ Edition Varanasi,Chaukhamba Bharati Acadamy 1993. Page no.-17.

8) Dr. Krushachandra Chunekar \& Dr. Gangasahay Pandeya, Bhavprakashnighantu of Sri bhavamisa, $\quad 9^{\text {th }} \quad$ Edition Varanasi,Chaukhamba Bharati Acadamy 1993. Page no.-15.

9) Dr. Krushachandra Chunekar \& Dr. Gangasahay Pandeya, Bhavprakashnighantu of Sri bhavamisa, $\quad 9^{\text {th }} \quad$ Edition Varanasi,Chaukhamba Bharati Acadamy 1993. Page no.-216.

10) Dr. Krushachandra Chunekar \& Dr. Gangasahay Pandeya, Bhavprakashnighantu of Sri bhavamisa, $\quad 9^{\text {th }} \quad$ Edition Varanasi,Chaukhamba Bharati Acadamy 1993. Page no.-219.

11) Dr. Krushachandra Chunekar \& Dr. Gangasahay Pandeya, Bhavprakashnighantu of Sri bhavamisa, $\quad 9^{\text {th }} \quad$ Edition Varanasi,Chaukhamba Bharati Acadamy 1993. Page no.-12\&13. 
12) Prof. Dr.Y.G. Joshi, Charak Samhita of Maharshi Agnivesha with Charakdatta commentary. $2^{\text {nd }}$ Edition. Pune. Vaidyamitra Prakashan-2005. Page No-425
13) Harishchandra Singh Kushwaha, Charaka samhita of Maharshi Agnivesh with Ayurved Dipika's Ayusi Hindi commentary. 2011. Editon, Varanasi, Chaukhamba orientalia 2011. Page No.-631. 